Annales Geophysicae (2001) 19: 1509-1522 C) European Geophysical Society 2001

\title{
Cluster PEACE observations of electrons during magnetospheric flux transfer events
}

\author{
C. J. Owen ${ }^{1}$, A. N. Fazakerley ${ }^{1}$, P. J. Carter ${ }^{1}$, A. J. Coates $^{1}$, I. C. Krauklis ${ }^{1}$, S. Szita ${ }^{1}$, M. G. G. T. Taylor ${ }^{1}$, \\ P. Travnicek ${ }^{1,2}$, G. Watson ${ }^{1}$, R. J. Wilson ${ }^{1}$, A. Balogh ${ }^{3}$, and M. W. Dunlop ${ }^{3}$ \\ ${ }^{1}$ Mullard Space Science Laboratory, University College London, Holmbury St. Mary, Dorking, Surrey, UK \\ ${ }^{2}$ Institute of Atmospheric Physics, The Academy of Sciences of the Czech Republic \\ ${ }^{3}$ Blackett Laboratory, Imperial College of Science, Technology and Medicine, Prince Consort Road, London, UK
}

Received: 17 April 2001 - Revised: 16 July 2001 - Accepted: 16 July 2001

\begin{abstract}
During the first quarter of 2001 the apogees of the Cluster spacecraft quartet precessed through midday local times. This provides the first opportunity for 4 spacecraft studies of the bow shock, magnetosheath and the dayside magnetopause current layer and boundary layers. In this paper, we present observations of electrons in the energy range $\sim 10 \mathrm{eV}-26 \mathrm{keV}$ made by the Plasma Electron And Current Experiment (PEACE) located just inside the magnetopause boundary, together with supporting observations by the Flux Gate Magnetometer (FGM). During these observations, the spacecraft have separations of $\sim 600 \mathrm{~km}$. This scale size is of the order or less than the typical size of flux transfer events (FTEs), which are expected to be observed following bursts of reconnection on the dayside magnetopause. We study, in detail, the 3-D configuration of electron populations observed around a series of enhancements of magnetosheath-like electrons which were observed within the magnetosphere on 2 February 2001. We find that individual spacecraft observe magnetic field and electron signatures that are consistent with previous observations of magnetospheric FTEs. However, the differences in the signatures between spacecraft indicate that these FTEs have substructure on the scale of the spacecraft separation. We use these differences and the timings of the 4 spacecraft observations to infer the motions of the electron populations and thus the configuration of these substructures. We find that these FTEs are moving from noon towards dusk. The inferred size and speed of motion across the magnetopause, in one example, is $\sim 0.8 R_{E}$ and $\sim 70 \mathrm{~km} \mathrm{~s}^{-1}$ respectively. In addition, we observe a delay in and an extended duration of the signature at the spacecraft furthest from the magnetopause. We discuss the implications of these 4 spacecraft observations for the structure of these FTEs. We suggest that these may include a compression of the closed flux tubes ahead of the FTE, which causes density and field strength enhancements; a circulation of open flux tubes within the FTE itself, which accounts for the delay in the arrival of the magnetosheath electron pop-
\end{abstract}

Correspondence to: C. J. Owen (cjo@mssl.ucl.ac.uk) ulations at locations deepest within the magnetosphere; and a possible trapping of magnetospheric electrons on the most recently opened flux tubes within the FTE.

Key words. Magnetospheric physics (magnetopause, cusp and boundary layers; solar wind-magnetosphere interactions)

\section{Introduction}

The magnetic reconnection process, as originally proposed by Dungey (1961), has been widely accepted as the primary mechanism for coupling mass and energy from the solar wind into the magnetosphere. Observations of accelerated flows at the magnetopause, initially reported by Paschmann et al. (1979), and the applicability of the magnetic field and plasma stress balance conditions for reconnected field lines at this boundary (Sonnerup et al., 1981), are accepted as strong circumstantial evidence of the occurrence of magnetic reconnection. In addition, Russell and Elphic (1978) reported a regularly occurring characteristic magnetic field signature of the flux transfer event (FTE), which is now widely accepted as a manifestation of transient reconnection. Statistical studies (Rijnbeek et al., 1984; Berchem and Russell, 1984; Southwood et al., 1986; Lockwood, 1991; Lockwood and Wild, 1993; Le et al., 1993; Kuo et al., 1995; Russell et al., 1995; Kawano and Russell, 1996, 1997) have shown that the occurrence rate of these events is enhanced during periods of southward interplanetary magnetic field (IMF), the conditions favourable for reconnection at the dayside magnetopause. These studies have also shown that the mean interval between FTE signatures is of the order of $8 \mathrm{~min}$. However, Lockwood and Wild (1993) showed that the distribution of these intervals has a mode value at $3 \mathrm{~min}$, with upper and lower decile values of 1.5 and $18.5 \mathrm{~min}$, respectively.

There have been many studies of the properties and structure of FTEs (e.g. Daly et al., 1981, 1984; Paschmann et al., 1982; Scholer et al., 1982; Rijnbeek et al., 1982, 1988; 
Sibeck and Siscoe, 1984; Saunders et al., 1984; Elphic and Southwood, 1987; Sonnerup, 1987; Farrugia et al., 1987, 1988; Southwood et al., 1988; Smith and Owen, 1992; Hubert et al., 1992; Jacob and Cattell, 1993; Le et al., 1999). Thomsen et al. (1987) presented the first detailed description of particle distributions within both magnetospheric and magnetosheath FTEs. These authors showed that FTEs contain plasma from both the magnetosphere and the magnetosheath. However, the distributions are modified compared to those found in the bounding regions. Klumpar et al. (1990) showed that the plasma composition within the FTE is also distinct, which is again interpreted as strong evidence of association with reconnection. Sibeck $(1990,1992)$ and Sibeck and Smith (1992) suggested that the FTE signature could be caused by a transient pressure pulse causing a temporary relocation of the observing spacecraft into the plasma depletion layer and magnetosheath. While it is undoubtedly true that this scenario mimics many of the gross features of an FTE, studies of the detailed properties (e.g. Lockwood, 1991; Smith and Owen, 1992; Elphic et al., 1994; Song et al., 1994) indicate that not all FTE-like signatures are consistent with the Sibeck $(1990,1992)$ picture, and it is thus appropriate to retain the reconnection model of their formation.

There have also been many observational studies of ionospheric signatures relating to FTEs occurring on the dayside magnetopause (e.g. Goertz et al., 1985; Southwood, 1987; Crooker and Siscoe, 1990; Elphic et al., 1990; Lockwood et al., 1990; Oieroset et al., 1996; Rodger and Pinnock, 1997; Provan et al., 1998). Again, many of these studies are interpreted in terms of a reconnection model for FTE formation. This is also true for a wide variety of modelling and simulation studies which examine the formation and structure of the individual reconnected magnetic flux tubes (e.g. Kan, 1988; Hesse et al., 1990; Lee et al., 1993; Ku and Sibeck, 1997; Nakamura and Scholer, 2000).

In this paper, we examine some early Cluster electron data recorded by the Plasma Electron and Current Experiment (PEACE) as the quartet of spacecraft moved outbound through the northern post-noon quadrant of the dayside magnetosphere on 2 February 2001. We include supporting observations from the magnetometer experiment FGM. In the next section, we briefly describe the instrumentation on the 4 spacecraft. In order to provide some context for the observations, we then describe the orbital configuration of the Cluster quartet, particularly with reference to the expected local orientations of the magnetopause boundary and magnetospheric magnetic field. We then describe the observations themselves. The PEACE instruments each recorded a series of transient bursts of magnetosheath-like electrons during the $40 \mathrm{~min}$ prior to the first extended excursion into the magnetosheath itself. FGM observed a bipolar signature in the component of the field normal to the nominal local magnetopause surface. These observations are consistent with the passage of a series of FTEs over the Cluster quartet. We examine two of these FTE signatures in some detail. Finally, we discuss the implication of these observations for the internal structure of magnetospheric FTEs, and propose one possible interpretation of these observations, before providing a summary and conclusions section.

\section{Instrumentation}

In this paper, we use data from the PEACE (Plasma Electron and Current Experiment) instruments from each of the four Cluster spacecraft, together with supporting observations from the Cluster FGM (Flux Gate Magnetometers) (Balogh et al., 1997, this issue).

The four identical PEACE instruments are designed to measure the electron velocity distribution in the vicinity of the spacecraft, each covering an energy range from $0.7 \mathrm{eV}$ to $\sim 30 \mathrm{keV}$ and detecting electrons arriving from all directions during a spacecraft spin (nominally $4 \mathrm{~s}$ ). Each PEACE instrument has two sensor heads, a Low- and a High-Energy Electron Analyser, LEEA and HEEA, respectively, which are mounted on opposite sides of the spacecraft. Thus, the instantaneous field-of-view of one sensor head is the same as that seen by the other sensor head a half spacecraft rotation period later. LEEA and HEEA differ only in geometric factor (HEEA admits more electrons than LEEA in an identical plasma). Note that each sensor samples the full $4 \pi$ steradians per spacecraft spin. The full energy range is divided into 88 logarithmically spaced levels. Each sensor is typically set up to sample an energy range of 60 contiguous levels. Both LEEA and HEEA can be used to sample any group of 60 or 30 levels within the full range of 88 levels. Thus, neither sensor can cover the full energy range. However, full coverage of the energy range is achieved by using both together. HEEA will normally cover the upper part of the energy range, since its larger geometric factor enables better measurements of the more diffuse electron population at higher energies. Note that in the energy ranges of the two sensors that overlap, we obtain $4 \pi$ steradian coverage every half spin. Each PEACE instrument makes an onboard calculation of the electron moments (for restricted energy ranges), and, with reference to the measurements made by FGM, selects a subset of the full 3-D data to form a pitch angle distribution. These two data products are transmitted to the ground during normal mode operation of the spacecraft.

The FGM instrument makes a measurement of the 3-D magnetic field vector. In this paper we use data at the spacecraft spin resolution (i.e. 4 s).

\section{Orbit and configuration}

In this paper, we are concerned with Cluster observations located just inside the magnetopause crossing on 2 February 2001. In order that the observations can be put into appropriate context, we first examine the orbit and the configuration of the Cluster tetrahedron. At the time of the observations, the spacecraft are located at around (5.9, 7.0, 9.2) $R_{E}$ in the GSE coordinate system and are moving outbound through the northern post-noon quadrant of the day- 
Cluster Configuration
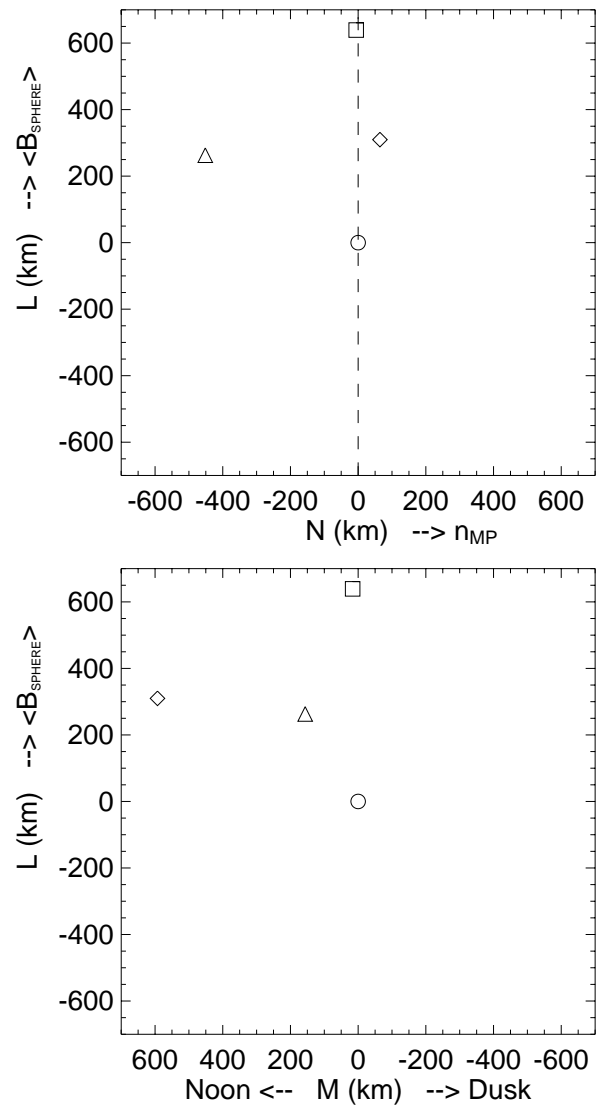

Boundary Normal Coordinates

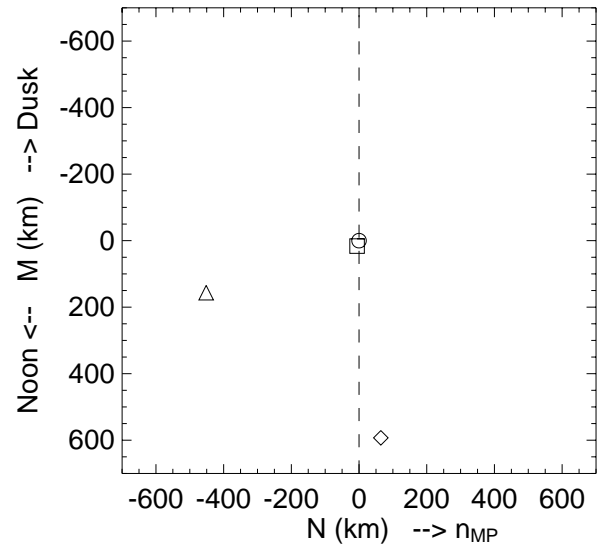

\author{
Cluster 1 \\ $\diamond \quad$ Cluster 2 \\ Cluster $3 @(5.86,7.04,9.17) R_{E}$ \\ $\triangle$ Cluster 4
}

Fig. 1. The configuration of the Cluster quartet at $\sim 14: 00$ UT on 2 February 2001, given in the boundary normal coordinate system determined from the Fairfield (1971) model magnetopause. The top left panel shows the projection of the spacecraft positions onto the $L N$ plane, the top right panel shows the projection onto the $M N$ plane and the bottom left panel shows the projection onto the $L M$ plane. The latter represents the plane of the magnetopause, the cross section of which is indicated by the dashed line on the upper two panels. Note that C1, C2 and C3 lie in a plane which is almost parallel to the magnetopause, with C2 located closest to the noon meridian. C4 lies deeper inside the magnetosphere. The spacecraft separations are of the order of $600 \mathrm{~km}$ at this time.

side magnetopause. We employ the Fairfield (1971) model of the magnetopause, scaled to the observed position, to determine an approximate boundary normal appropriate to these observations. We then employ a boundary normal coordinate system $(L M N)$ in which the $N$-direction points outward along the boundary normal, the $L$-direction is parallel to the projection of the dipole axis onto the local plane of the boundary, such that it points along the expected direction of the magnetospheric field located just inside the boundary. Finally, the $M$-direction makes up the right-hand set. In this case, the unit vectors $\boldsymbol{l}, \boldsymbol{m}, \boldsymbol{n}$ are given by $(-0.582,-0.268$, $0.768),(-0.524,-0.845,0.103)$ and $(0.621,0.463,0.632)$, respectively. The normal direction thus points in the positive GSE $X, Y$ and $Z$ directions, which is appropriate for the northern post-noon magnetopause. Note also that although this boundary normal is determined from a model magnetopause, it can be shown that it is consistent with the results of both minimum variance and 4 spacecraft timing analyses applied at the real magnetopause crossings observed during the interval of interest.

We now rotate the spacecraft separation vectors into this $L M N$ coordinate system, so that the configuration of the quartet relative to the boundary can be visualised. This is displayed in Fig. 1, which shows the spacecraft positions (relative to Cluster 3, the reference spacecraft, located at the origin) in the $L N$ (top left), $M N$ (top right) and $L M$ (bottom left) planes. The latter is the expected plane of the magnetopause, with noon local times towards the left of the figure, dusk to the right of the figure, with increasing northern latitudes towards the top. From this panel, it is clear that Cluster spacecraft 1, 2 and 3 (henceforth $\mathrm{C} 1, \mathrm{C} 2, \mathrm{C} 3$ ) form an approximate equilateral triangle with sides $\sim 600 \mathrm{~km}$ long. $\mathrm{C} 2$ is located closest to the noon meridian, with $\mathrm{C} 1$ and $\mathrm{C} 3$ located at similar $M$ values towards dusk. C4 appears in the centre of the triangle. However, as it is clear from the two upper plots, which show the separation in the 2 planes normal to the expected magnetopause boundary, this spacecraft is located somewhat Earthward of the other three. C1, C2 
and $\mathrm{C} 3$ have small separations in the direction normal to the boundary. Since $\mathrm{C} 1$ and $\mathrm{C} 3$ also have small separations in the $M$ direction, these two spacecraft are nominally closely aligned along the same flux tubes while within the magnetosphere. Note that with this configuration, boundaries moving Earthward should pass C1, C2 and C3 ahead of C4. Magnetospheric flux tubes moving from noon towards dusk, in a direction normal to their expected magnetic field vector and parallel to the magnetopause, should appear first at $\mathrm{C} 2$ and last, but almost simultaneously at $\mathrm{C} 1$ and $\mathrm{C} 3$. We now examine the PEACE and FGM observations made on this day.

\section{Observations}

Figure 2 shows an overview of the PEACE electron and FGM magnetic field data taken between 13:00 UT and 15:30 UT on 2 February 2001. During this time, the Cluster spacecraft were moving outbound through the northern afternoon sector of the dayside magnetosphere. Each of the top 4 panels of the figure shows an overview spectrogram of the PEACE data from each of the four Cluster spacecraft $(\mathrm{C} 1$ to $\mathrm{C} 4$ from top to bottom, respectively) for this period. The energy range on the vertical axis is from $10 \mathrm{eV}$ to $30 \mathrm{keV}$ and the differential number flux of the electrons is represented by the colour bar on the right side of the figure. These spectrograms are derived from the pitch angle distribution calculated on board the spacecraft, and have a basic time resolution of $4 \mathrm{~s}$, which is the spin period of each of the 4 spacecraft. Note that in this work, we have only applied preliminary ground calibrations to the PEACE data, such that there may be minor differences in the relative fluxes between spacecraft. The lower 4 panels show the GSE magnetic field components from the $\mathrm{C} 1$ spacecraft for this 2.5 hour period. (Note that on this time scale, the data from the other 3 spacecraft are essentially identical to this and thus not shown.) From top to bottom, we show the $B_{Z}, B_{Y}, B_{X}$ and the field magnitude, $B_{\text {mag }}$, respectively.

At the start of the period shown in Fig. 2, the four Cluster spacecraft are located well within the magnetosphere, as indicated by the moderate fluxes of electrons at higher energies. (Note that the most intense fluxes in these regions occurring at the lowest $(<20 \mathrm{eV})$ energies in these plots are associated with photoelectrons and secondary electrons emitted from the spacecraft themselves and are, therefore, ignored in the subsequent analysis.) At $\sim 13: 30 \mathrm{UT}$, the $B_{Z}$ component in the 5 th panel of the figure turns positive. From this time until $\sim 14: 00 \mathrm{UT}, B_{Z}$ continues to increase, while the $B_{X}$ and $B_{Y}$ components are negative at about $-8 \mathrm{nT}$ and $-16 \mathrm{nT}$, respectively. This field configuration within the magnetosphere is consistent with the spacecraft position in the northern afternoon flank quadrant of the magnetosphere, and indicates that the spacecraft is located equatorward, sunward and duskward of the northern cusp. The electron and $\boldsymbol{B}$-field data thus indicate that the spacecraft are located on closed dayside field lines during this period. Towards the end of the interval shown in Fig. 2, the quartet makes two excur- sions across the magnetopause and into the magnetosheath ( $\sim$ 14:38-15:00 UT and $\sim 15: 10-15: 30$ UT). Each spacecraft observes much colder and denser space plasma electron populations in this region. The magnetic field is more variable, but on average, points northward and antisunward in the first interval before switching to a predominantly duskward orientation in the second interval.

In this paper, we are concerned particularly with the boundary region between these two plasma populations, which is observed during the $40 \mathrm{~min}$ prior to the first magnetopause crossing at $\sim 14: 38$ UT. During this period, the magnetic field vector fluctuates in both magnitude and direction, although the $B_{Z}$ component continues to increase, indicating that the average direction tilts increasingly northward as the magnetopause is approached. The PEACE data for this transition interval are shown in more detail in Fig. 3. This figure has the same format as the upper 4 panels of previous figure. The first magnetopause crossing is marked by the red arrow at the bottom of the figure. This marks a clear transition of the spacecraft into the cold, dense plasma of the magnetosheath region. However, in the $40 \mathrm{~min}$ prior to this crossing, each of the four Cluster spacecraft observe transient bursts of magnetosheath electrons, each lasting for a period of $\sim 1 \mathrm{~min}$. These are marked by the series of blue arrows at the bottom of the figure. Note that the event occurrence interval is of the order of $4-6 \mathrm{~min}$. This interval is characteristic of the typical occurrence rate of FTEs (Lockwood and Wild, 1993). For the remainder of this paper, we will concentrate in detail on observations of two of these FTE-type signatures, the first event observed at 14:06 UT, and the third event centred on 14:19 UT. Figure 4 shows a close-up of the first of these events, covering the 4 min from 14:04-14:08 UT, in the same format as the previous figure. On these time scales, the differences between the observations made by the individual spacecraft become more apparent. C1, C2 and C3 observe an abrupt onset of magnetosheath electrons, with the signature observed at $\mathrm{C} 2$ slightly ahead of $\mathrm{C} 1$ and $\mathrm{C} 3$. An enhancement in the flux of magnetosheath-like electrons is also observed by $\mathrm{C} 4$, located further into the magnetosphere. However, this enhancement appears more gradual, and the signature duration appears to be somewhat longer at this spacecraft. In addition to the enhancement in magnetosheath electrons, there is some indication of a dropout in the fluxes of higher energy electrons during these events. These features are clearer in the upper 4 panels of Fig. 5, which shows density moments from the 4 spacecraft for this interval (top 4 panels, $\mathrm{C} 1$ to $\mathrm{C} 4$, respectively). Again, note that these densities were derived on board the spacecraft with the initial ground calibration in place. Hence, there are some differences in the absolute density values returned from each spacecraft. In these panels, we plot the densities of electrons in two energy ranges. The black line in each panel shows densities of electrons in the energy range of $34 \mathrm{eV}$ to $1.2 \mathrm{keV}$, while the red line shows the (relatively much lower) densities of electrons with energies of 1.2 to $26 \mathrm{keV}$. The scales relevant to these two density ranges are shown on the left and right vertical axes respectively. This approach has two advantages. First, the lower 

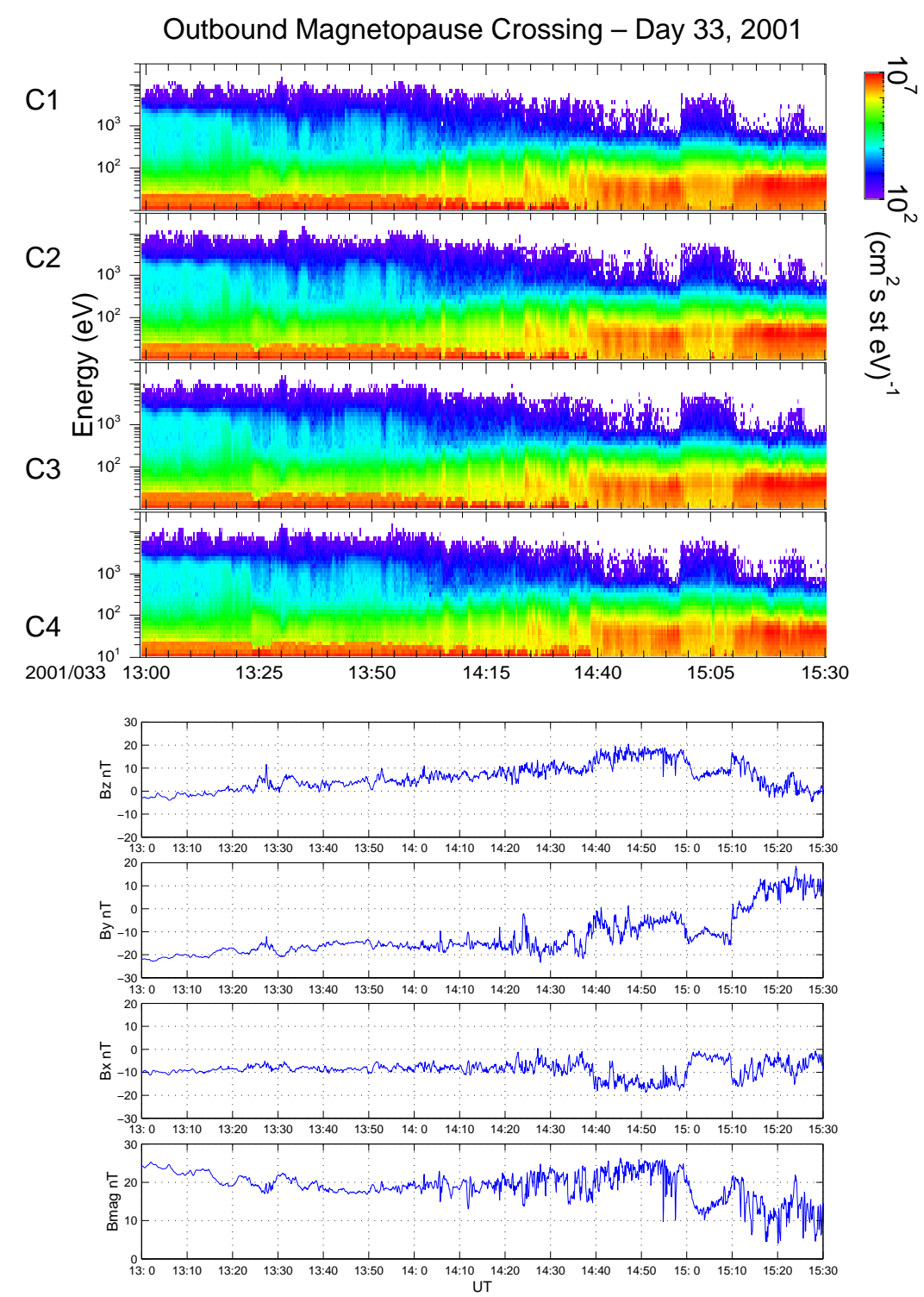

Fig. 2. An overview of the Cluster PEACE and FGM data for the period from 13:00-15:30 UT on 2 February 2001. The top 4 panels show energy-time spectrograms for electrons in the energy range of $10 \mathrm{eV}-30 \mathrm{keV}$ for $\mathrm{C} 1$ to $\mathrm{C} 4$, respectively. The lower 4 panels show the GSE components of the magnetic field vector observed by the C1 FGM instrument. During this period, the Cluster quartet moved from closed flux tubes within the northern post-noon quadrant of the dayside magnetosphere and equatorward of the cusp, through a boundary region and into the magnetosheath. The magnetosphere is populated by an sparse, energetic electron population, while the magnetosheath contains a cold dense plasma.

range covers the expected energies of electrons of magnetosheath origin, while the upper range covers the expected energy range of magnetospheric electrons. Second, the lower range represents the overlap region of the HEEA and LEEA sensors on each spacecraft, and thus densities in this range are determined twice per spin, i.e. with $2 \mathrm{~s}$ resolution, rather than the standard $4 \mathrm{~s}$ spin resolution. The lower panels of Fig. 5 show the magnetic field components observed at each of the 4 spacecraft in the boundary normal coordinate system. In these panels, magnetic field data returned from $\mathrm{C} 1$ is shown as the black trace, $\mathrm{C} 2$ is red, $\mathrm{C} 3$ is green and $\mathrm{C} 4$ is magenta. The first of these panels shows the magnetic field vec- tors, $B_{L}$, observed in the model $L$-direction. Below this, we show the $B_{M}$ traces, while in the penultimate panel, we show the $B_{N}$ components of the field in the model magnetopause normal direction. The bottom panel shows the magnetic field strength at each of the 4 spacecraft. These magnetic field data are presented at $4 \mathrm{~s}$ resolution.

In the upper panels of Fig. 5, the sharp rise in the density of magnetosheath-like electrons at $\mathrm{C} 1, \mathrm{C} 2$ and $\mathrm{C} 3$ is evident in the $10 \mathrm{~s}$ period following 14:05:34 UT. It is clear that the enhancement occurs at $\mathrm{C} 2$ a few seconds prior to $\mathrm{C} 1$ and $\mathrm{C} 3$. The enhancement at $\mathrm{C} 4$, deeper in the magnetosphere occurs more gradually, with the peak in the enhancement occurring 


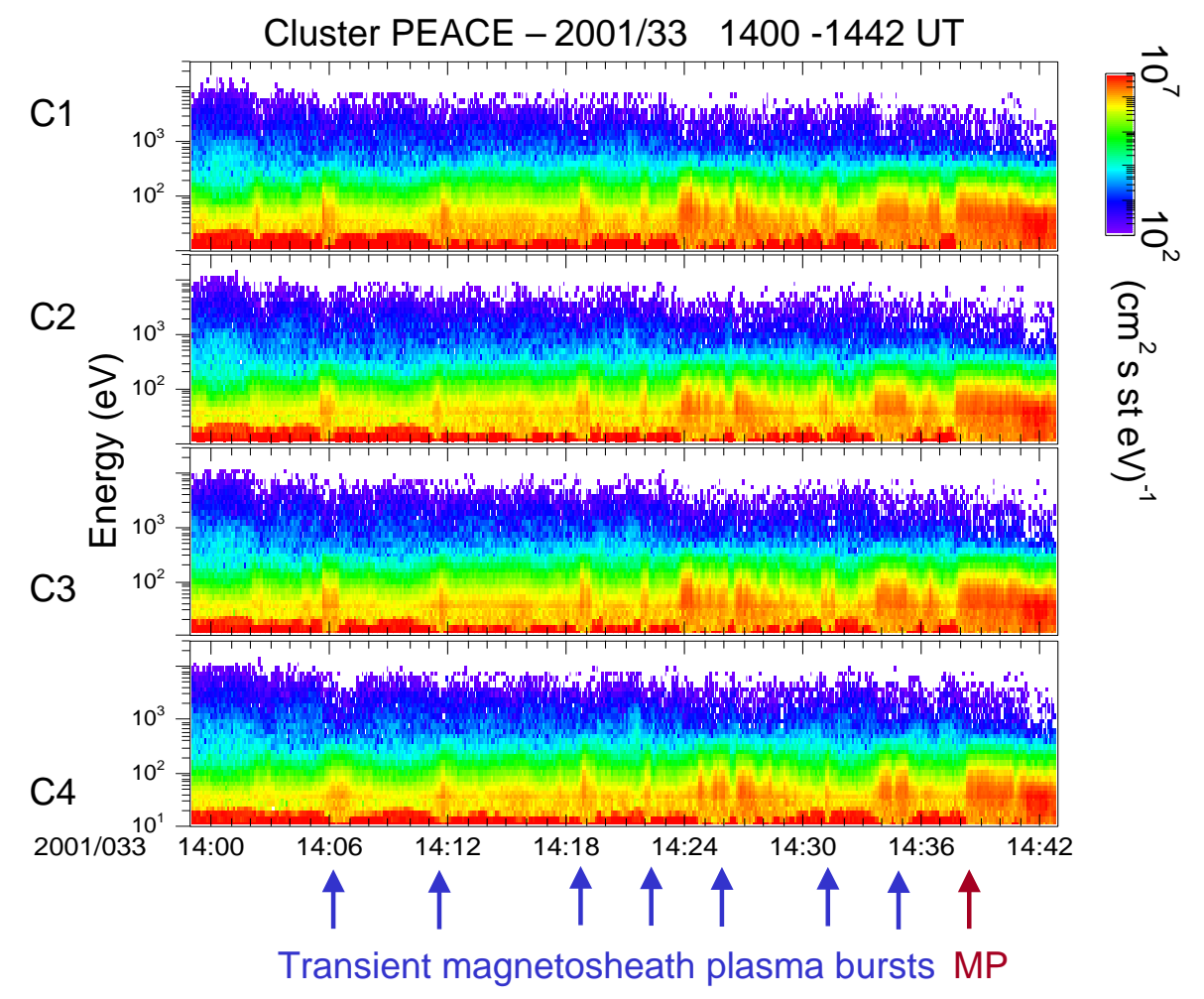

Fig. 3. PEACE energy-time spectrograms for the period from 14:00-14:42 UT on 2 February 2001. As the spacecraft approach the magnetopause, indicated by the red arrow at the bottom of the figure, a series of transient bursts of magnetosheath-like electrons are observed by each of the PEACE instruments (blue arrows). These occur every 4-6 min, which is consistent with the occurrence rate of magnetospheric flux transfer events.

some $40 \mathrm{~s}$ after the peaks observed at the other 3 spacecraft. In addition, $\mathrm{C} 4$ observes only a single peak in the enhancement, while each of the other three spacecraft observe a double peaked structure. This clearly indicates structure within the event on the spatial scale of the spacecraft separation $(\sim 600 \mathrm{~km})$. Note also that the decay in the enhancement at $\mathrm{C} 1, \mathrm{C} 2$ and $\mathrm{C} 3$ is more gradual than the onset, and the time order for the occurrence of this decay is the same as the onset, with $\mathrm{C} 2$ leading $\mathrm{C} 1$, which slightly leads $\mathrm{C} 3$. Again the decay at $\mathrm{C} 4$ is delayed and more extended than that observed at the other 3 spacecraft. Considering now the behaviour of the electrons with energies characteristic of the magnetospheric population (as indicated by the red line in each of the 4 panels), we see that the density of these particles is indeed lower during the enhancement of magnetosheath electrons. However, both before and after those enhancements, there is clear evidence of a short-lived $(\sim 10-20 \mathrm{~s})$ enhancement of magnetospheric electrons at C1, C2 and C3. Such an enhancement is also evident at $\mathrm{C} 4$ prior to the appearance of the magnetosheath electrons, but is not observed at the end of that event, at least on the time scale shown in the figure. However, compressions in the wake of FTEs have also been reported by Hubert et al. (1992). The lower resolution magnetic field data in the lower panels of the figure give support to the timing order determined from the $2 \mathrm{~s}$ PEACE overlap densities described above. Note also that immedi- ately prior to the observation of magnetosheath electrons at each spacecraft, the corresponding magnetic field shows an increase in the strength, and then a reduction during the observation of magnetosheath electrons. The normal component of the magnetic field, $B_{N}$, has a significant background component of $\sim 8 \mathrm{nT}$. However, the observation of magnetosheath electrons at $\mathrm{C} 1, \mathrm{C} 2$ and $\mathrm{C} 3$ is associated with first a positive deflection in $B_{N}$, and then a more moderate but temporally extended negative deflection at each spacecraft (the black, red and green traces, respectively). Such bipolar signatures are consistent with the expected magnetic signature within an FTE (e.g. Russell and Elphic, 1978). Again, these signatures are seen less clearly in the data from C4 (magenta trace), where the peak positive deflection occurs $\sim 50 \mathrm{~s}$ after that at $\mathrm{C} 1$ and $\mathrm{C} 3$. However, there is little evidence of a negative deflection at this spacecraft.

We now look at the third FTE-like event observed during this period, which was centred on 14:19 UT. (We do not consider the second FTE since a data gap occurred at C4 in the middle of this event. Neither will we consider the later enhancements as they are either qualitatively similar to the first and third events or exhibit a considerably more complicated signature.) The spectrograms for this event are shown in Fig. 6, in the same format as Fig. 4. In this case, a relatively abrupt onset of the enhancement of magnetosheath electrons occurs at all 4 spacecraft. However, a clear dou- 


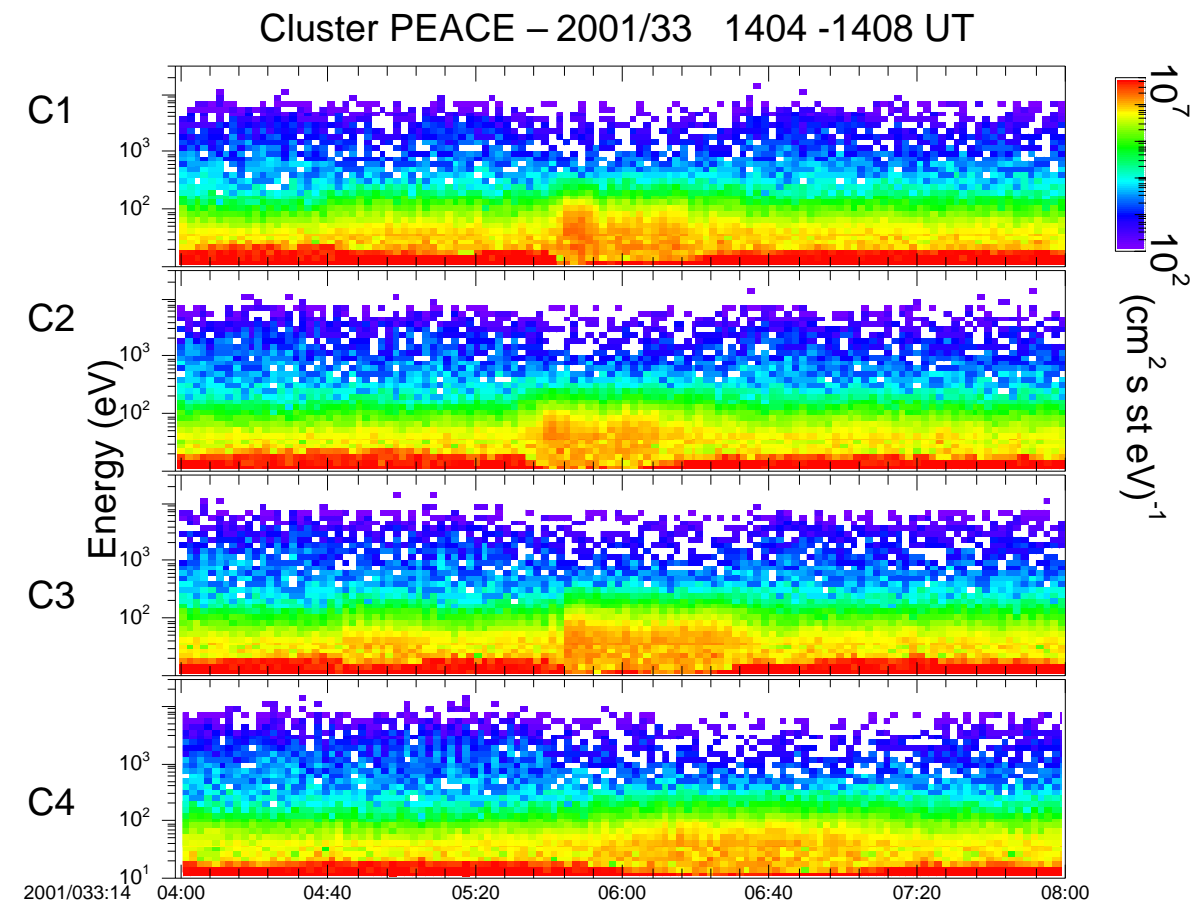

Fig. 4. PEACE energy-time spectrograms for the period from 14:04-14:08 UT on 2 February 2001, a period covering the observation of a burst of magnetosheath electrons. On this reduced time scale, the differences between the signatures observed at the individual spacecraft are apparent. C1, C2 and C3, located closest to the magnetopause, observe a sharp onset, with the C2 signature leading the other 2 by a few seconds. Conversely, the $\mathrm{C} 4$ signature is significantly delayed, and has a longer duration but is less intense and less structured.

ble peak signature is evident in $\mathrm{C} 1, \mathrm{C} 2$ and $\mathrm{C} 3$, which is not obvious in the $\mathrm{C} 4$ data. This again indicates structure within the FTE over a spatial scale of $<600 \mathrm{~km}$. As in the previous event, the signature in $\mathrm{C} 2$ precedes that at $\mathrm{C} 1$ and $\mathrm{C} 3$, with a further delay to the signature at $\mathrm{C} 4$. Note also that during the period from 14:19:44-14:20:04 UT, each spacecraft observes a brief, weak signature of magnetosheath-like electrons in which the signature at $\mathrm{C} 4$ now precedes that at $\mathrm{C} 1$ and C3. Again, each of these structures are clearer in the density moments for this event, shown in Fig. 7. The top 4 panels of Fig. 7, in the same format as Fig. 5, indicate that the main enhancement of magnetosheath-like electrons is clearly seen at all 4 spacecraft, centred on 14:19 UT, with a double peaked structure again observed at $\mathrm{C} 1, \mathrm{C} 2$ and $\mathrm{C} 3$. Comparing the timings of the steepest gradients, it is again clear that both the onset and decay of this magnetosheath electron signature occurs first at $\mathrm{C} 2$, then almost simultaneously at $\mathrm{C} 1$ and $\mathrm{C} 3$, and finally at $\mathrm{C} 4$. Given the configuration of the Cluster tetrahedron discussed above, it appears that both the leading and trailing boundaries of this electron signature are moving duskward and into the magnetosphere. It is also clear that a brief enhancement in magnetospheric electrons is again observed both immediately before and after this main region of magnetosheath electrons. However, there is a dropout of these electrons during the observation of the magnetosheath electrons. Note also the short duration pulse of magnetosheath-like electrons occurring at each spacecraft after the trailing edge of the enhancement of mag- netospheric electrons. This moderate transient enhancement in the magnetosheath electrons might have been considered unimportant, if it were not for the fact that the timing of the signatures of this feature differs from those observed previously. In this case, both the leading and trailing edges of the enhancement arrive at $\mathrm{C} 2$ before $\mathrm{C} 1$ and $\mathrm{C} 3$, as before. However, the enhancement is also observed at $\mathrm{C} 4$ prior to $\mathrm{C} 3$, with the $\mathrm{C} 1$ signature observed last. Although this order remains consistent with a structure moving from noon towards dusk, this is the first and only indication observed during this event of a motion back towards the magnetopause .

The lower resolution magnetic field data shown in the lower panels of Fig. 7 again support the timing order of the FTE signature at each of the 4 spacecraft. In addition, there is again a moderate enhancement of the field magnitude prior to the arrival of the magnetosheath electrons at each spacecraft. In this example, all 4 spacecraft observe a brief but clear positively directed deflection in the normal component, $B_{N}$, before a more extended negative excursion. Note that the peak negative values in these bipolar signatures do not occur until $\sim$ 14:20 UT or later, i.e. generally after the second minor pulse of magnetosheath electrons is observed by the corresponding PEACE instrument. Consequently, the magnetic signature of the FTE extends beyond the combined extent of the two enhancements of magnetosheath electrons. 

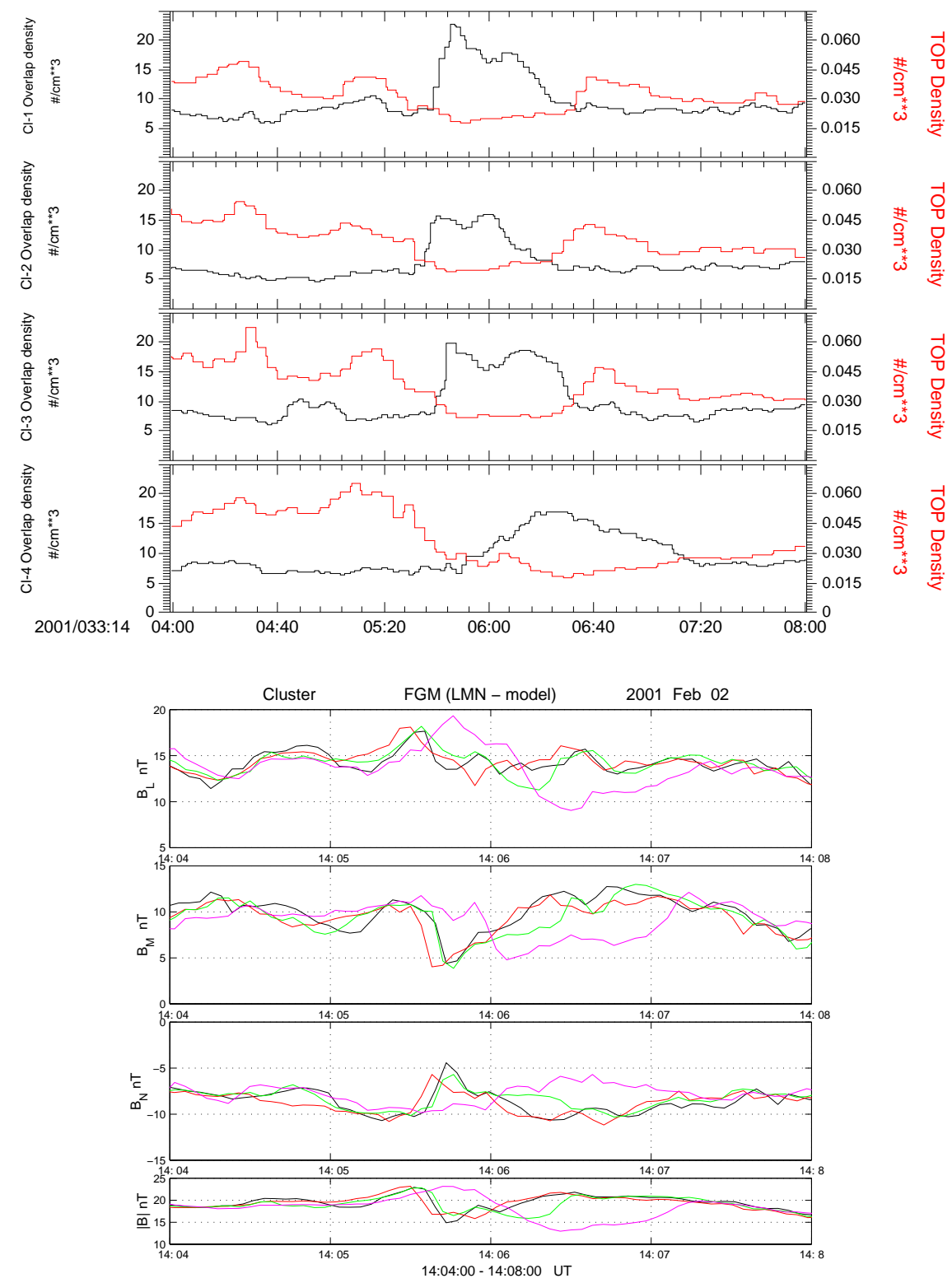

Fig. 5. (a) (Top 4 panels) Four spacecraft PEACE density moments for the period shown in Fig. 4 for electrons in the energy range of $34 \mathrm{eV}-1.2 \mathrm{keV}$ (black traces) and 1.2-26 keV (red traces). The former covers the typical energy range of magnetosheath electrons, the latter that of magnetospheric electrons. The rapid enhancement of magnetosheath electrons at $\mathrm{C} 1, \mathrm{C} 2$ and $\mathrm{C} 3$ is clearly seen. In addition, enhancements in the magnetospheric electrons are seen on either side of the observation of magnetosheath electrons, with a dropout in the middle. C4 sees a delayed enhancement of magnetosheath electrons, and no enhancement of magnetospheric electrons on the trailing edge. (b) (Lower 4 panels) The $L M N$ magnetic field components and field strength observed by the 4 Cluster spacecraft (C1 - black, C2 - red, C3 - green, C4 - magenta trace) during this period. The field strength is slightly enhanced at each spacecraft immediately prior to the arrival of the magnetosheath electrons. The $B_{N}$ component shows a brief positive and then more extended negative deflection, which is consistent with the expected signature of a magnetospheric FTE.

\section{Discussion}

The observations discussed above are consistent with the Cluster quartet encountering a series of magnetospheric FTEs as it approaches the northern post-noon magnetopause on 2 February 2001. The inter-event timings, of the order of 4 to $8 \mathrm{~min}$, are consistent with previous observations of FTEs (e.g. Rijnbeek et al., 1984; Lockwood and Wild, 1993).
Considering the 2 events discussed in detail here, the observation of bipolar signatures in each of the FGM data sets is also consistent with this interpretation (Russell and Elphic, 1978). In addition, the PEACE signature observed by the individual spacecraft represents a brief pulse of magnetosheath electrons associated with a dropout of the higher energy magnetospheric electrons. This is also consistent with previous reports of FTE structure (e.g. Smith and Owen, 1992). In 


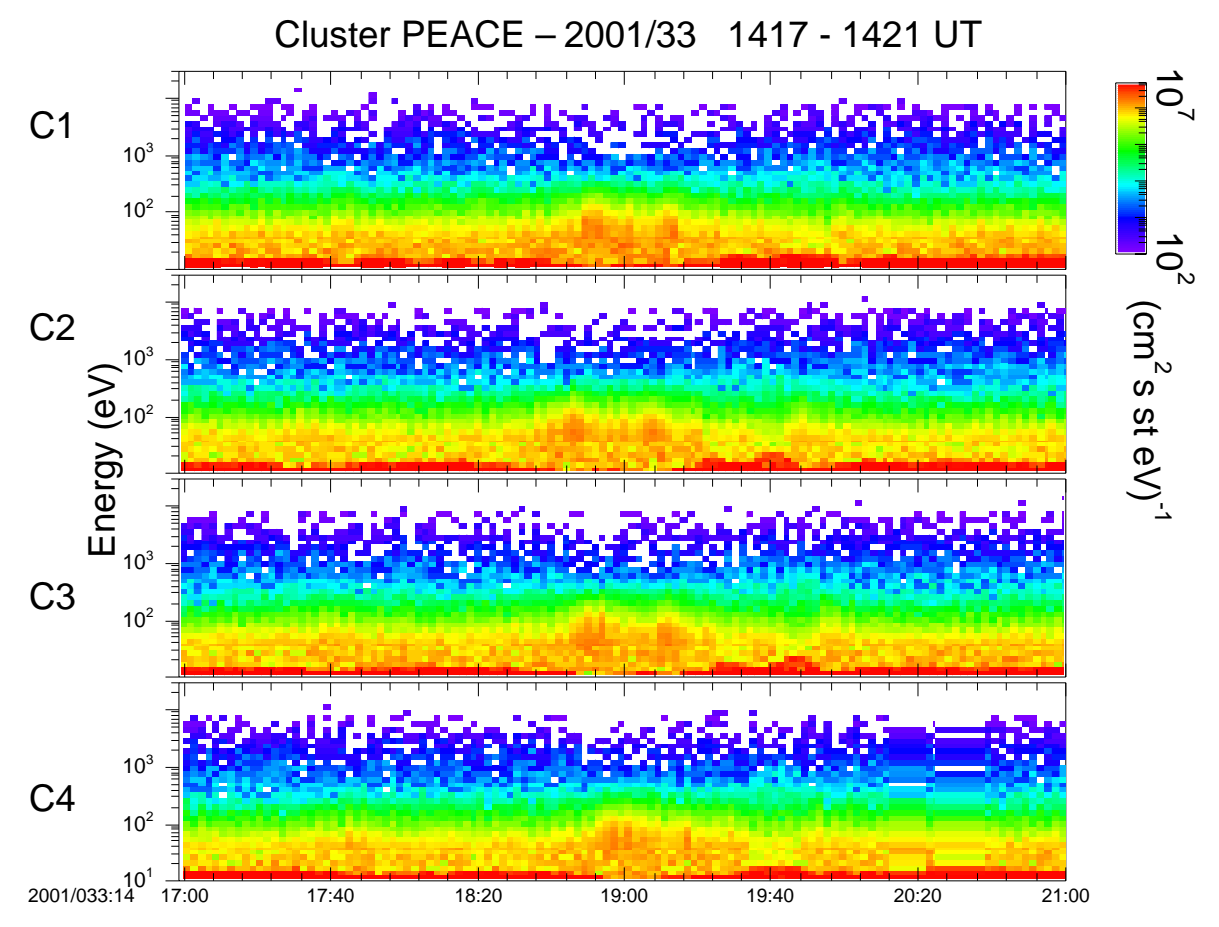

Fig. 6. As Fig. 4, for the period from 14:17-14:21 UT. An enhancement in magnetosheath electrons is again evident in this period. The onset is relatively rapid at all 4 spacecraft, but as in the previous example, $\mathrm{C} 4$, located deeper into the magnetosphere, observes a less structured population.

the observations presented here, enhancement of magnetospheric electrons outside the main regions of magnetosheath electrons also occur at most spacecraft, and are associated with a modest enhancements of the magnetic field strength. This may suggest a compression of the flux tubes associated with the magnetospheric electrons, perhaps as a result of a snowplow effect as these structures move through the magnetospheric flux tubes. Of more interest here are the differences observed between spacecraft, both in terms of the timing of the signatures and the structure within them. In both the events discussed above, it is clear that there are electron structures within the FTEs that have spatial scales smaller than or comparable to the inter-spacecraft separations, which are of the order of $600 \mathrm{~km}$ in this case. In general, C1, C2 and $\mathrm{C} 3$, which are the 3 spacecraft lying in a plane approximately parallel to the expected magnetopause surface, observe qualitative the same $B$-field and electron signatures. In particular, there are fairly clear double peaked structures in the magnetosheath electrons observed at these 3 spacecraft in both FTE cases presented here. In contrast, C4, lying some $500 \mathrm{~km}$ further Earthward of that plane, observes a qualitatively different signature. In particular, in the first event described above, the signature recorded at $\mathrm{C} 4$ is delayed, is weaker and is longer in duration than that observed at the 3 spacecraft closer to the magnetopause. This implies that the structure is more diffuse at greater distances from the magnetopause. Note also that the onset and decay signatures in the first event occur at the 4 spacecraft in the same temporal order, namely first at $\mathrm{C} 2$, which is closest to noon, then at ap- proximately the same time at $\mathrm{C} 1$ and $\mathrm{C} 3$, which are located further duskward and aligned on the same magnetic meridian, and then finally at $\mathrm{C} 4$, deeper into the magnetosphere. This timing order is consistent with both the leading and trailing edges of a structure moving from noon towards dusk and into the magnetosphere. The apparent motion also appears to be closely perpendicular to the expected direction of the background magnetospheric field, as evidenced by the near simultaneous signature at $\mathrm{C} 1$ and $\mathrm{C} 3$. There is no evidence of a boundary moving back out towards the magnetopause in this event, as might be expected from a cylindrical model of a flux tube moving around the magnetopause, or a boundary wave on the magnetopause.

The PEACE and FGM data for the second event discussed above shows many of the same characteristics as the first. One significant difference is that the PEACE signature observed at $\mathrm{C} 4$, deepest into the magnetosphere, is not as weak or as diffuse as the earlier example, perhaps as a result of the quartet being located closer to the magnetopause for this event. In addition, there appears to be a second enhancement of magnetosheath electrons on the trailing edge of the structure. This second enhancement is, however, contained within the envelope of the bipolar signature in the normal component of the magnetic field observed by the FGM instruments. Since each of the leading/trailing edges of the two pulses of magnetosheath electrons that occur during the second event (Fig. 7) are discernable at all 4 spacecraft, a more quantitative analysis of these 4 boundary motions can be performed. We make the simplifying assumption that each of these bound- 

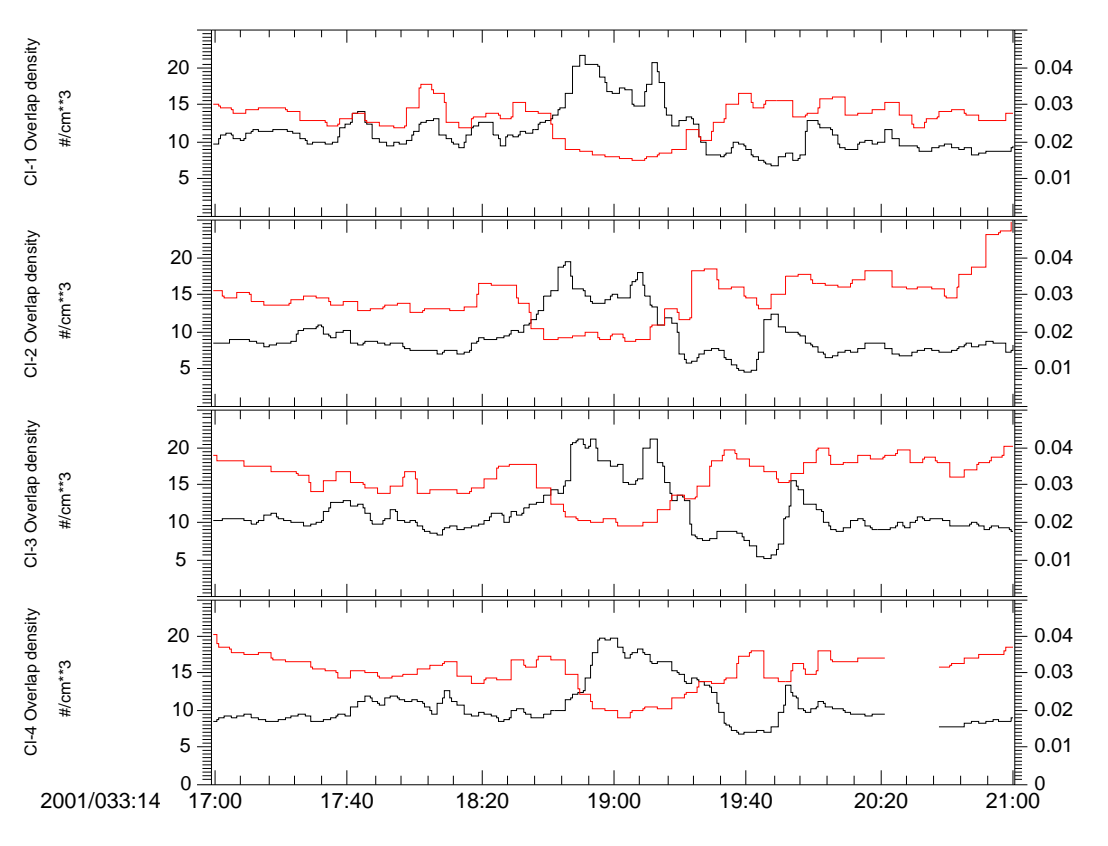

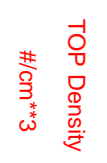

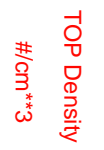
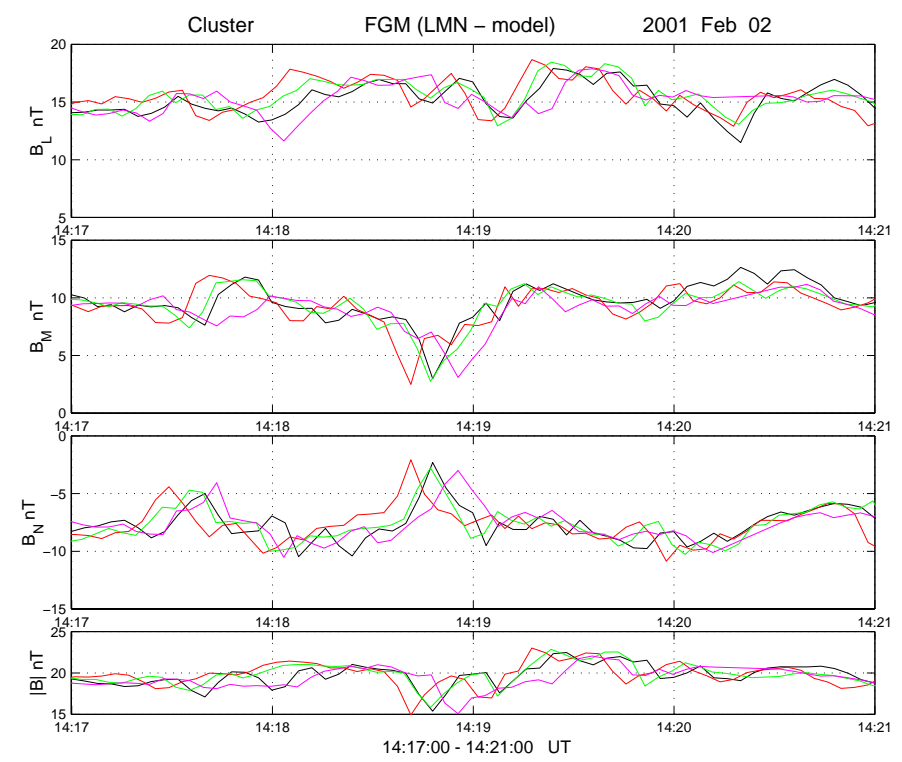

Fig. 7. As Fig. 5, for the period from 14:17-14:21 UT. (a) Enhancements in the density of magnetosheath-like electrons are observed at all 4 spacecraft approximately centred on 14:19 UT. Brief enhancements in the magnetospheric electrons are seen both before and after this magnetosheath electron population, with a dropout in between. A second, less dense population of magnetosheath electrons is observed at all 4 spacecraft in the period from 14:19:40-14:20:00 UT. (b) The magnetic field components and field strength for this period. The $B_{N}$ component again shows a bipolar signature, with a brief positive deflection followed by a more extended negative deflection.

aries can be approximated to a planar structure, and that each moves at uniform speed between the first and the last observations. Under these assumptions, the timing of the signatures at 4 points is sufficient to determine the orientation of the boundary and the speed of its motion in the boundary normal direction. Note that there is an uncertainty of $2 \mathrm{~s}$ in the timing of the observations of these boundaries due to the time resolution of the PEACE instrument over the energy range used. Since some of the time delays are also only a few seconds, there may be fairly large errors, particularly in the boundary speeds. The direction information is more robust as this is controlled primarily by the relative order of the signatures at the 4 spacecraft, rather than small changes in the magnitude of the inter-spacecraft delays.

The results of this analysis for the event shown in Figs. 6 and 7 are summarized in Fig. 8. In this figure, we show the orientation of the 4 boundaries in the $M N$ plane, i.e. the plane normal to the nominal direction of the magnetospheric field. Recall that vectors in the negative $M$ direction correspond to motion from noon local time towards dusk, while vectors in the negative $N$ direction represent motions towards the Earth or away from the magnetopause while the 
spacecraft is located within the magnetosphere. In this figure, we have translated the time intervals between the boundaries into an approximate spatial separation to give an indication of the spatial structures convecting past the spacecraft. The leading edge of the first enhancement of magnetosheath electrons is thus shown on the right side of the figure (i.e. furthest duskwards). As anticipated from the timing of the signatures, the normal to this boundary points duskward and Earthwards, thus making an angle of $40^{\circ}$ to the expected magnetopause surface. The associated speed of the boundary in this normal direction is $57 \mathrm{~km} \mathrm{~s}^{-1}$. Note also that for this boundary, the analysis returns a very small $\left(\sim 0 \mathrm{~km} \mathrm{~s}^{-1}\right)$ component of velocity in the $L$ direction, confirming that this surface moves perpendicular to the nominal magnetospheric field direction. Similar analysis for the trailing edge of the first enhancement of magnetosheath electrons returns a similar result, rather than a boundary moving back outwards, as might have been expected if this were a simple cylindrical intrusion of magnetosheath plasma convecting across the magnetopause. This boundary makes an angle of $47^{\circ}$ to the negative $M$ axis in the plane of Fig. 8, and has an associated velocity of $44 \mathrm{~km} \mathrm{~s}^{-1}$. This velocity also includes only a small component $\left(\sim 10 \mathrm{~km} \mathrm{~s}^{-1}\right)$ of the normal velocity in the positive $L$ direction, such that again this boundary is moving largely perpendicular to the nominal magnetospheric field. Turning now to the timings associated with the second, more minor enhancement in magnetosheath electron fluxes, we see the first evidence of material moving back towards the magnetopause. Analysis of the leading edge of this enhancement shows that this boundary moves duskward and outwards, such that the normal forms an angle of $20^{\circ}$ to the negative $M$ axis in the $M N$ plane. The associated velocity is $\sim 49 \mathrm{~km} \mathrm{~s}^{-1}$, although in this case, this includes a significant component of velocity $\left(\sim 28 \mathrm{~km} \mathrm{~s}^{-1}\right)$ in the positive $L$ direction. Finally, the trailing edge of this enhancement also moves duskward and outward, with an angle of $56^{\circ}$ to the negative $M$ axis and a velocity of $\sim 47 \mathrm{~km} \mathrm{~s}^{-1}$. The $L$ component of this velocity is $18 \mathrm{~km} \mathrm{~s}^{-1}$. In these two cases, the motion is predominantly perpendicular to the nominal orientation of the magnetospheric field, with velocity normals tilted only slightly into the positive $L$ direction. Each of the velocities determined above can be associated with a velocity of the structure along the expected direction of the magnetopause. The average of these boundary parallel velocities, which could be considered the convection speed of the entire FTE structure across the magnetopause, is $70 \mathrm{~km} \mathrm{~s}^{-1}$, as indicated in the figure. Applying this velocity to the observed duration of the event implies that the size of the structure is of the order of $0.8 R_{E}$ in the direction parallel to the magnetopause surface, as also indicated in the figure.

In Figs. 9a and b, we postulate a possible interpretation of these PEACE electron observations and associated FGM data. We assume that the observation of the magnetosheath electrons is associated with a reconnected magnetic flux tube in motion across the magnetopause. We show the cross section of this structure in the $M N$ plane and in the rest frame of the structure. The magnetopause is represented by the dashed

\section{Orientation of Observed Boundaries in NM Plane}

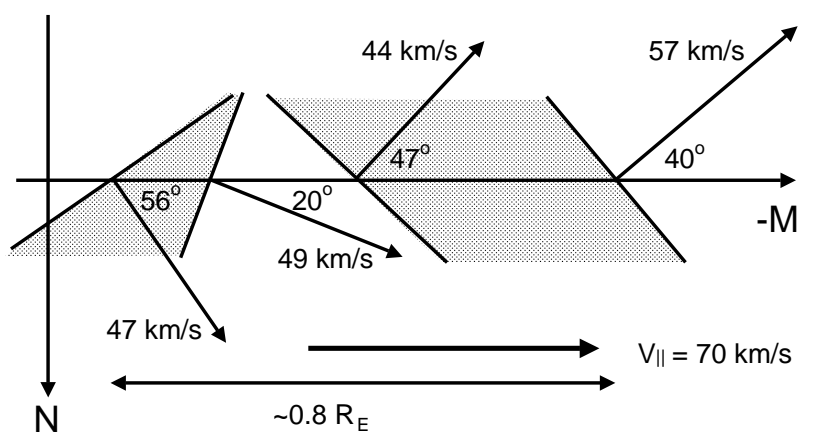

Sheath Plasma

Fig. 8. A summary sketch of the inferred orientation and motion of the observed boundaries of magnetosheath electron populations shown in Fig. 7. The leading edge of the first, major enhancement, represented on right, moves duskward and Earthward at $57 \mathrm{~km} \mathrm{~s}^{-1}$. Somewhat unexpectedly, the trailing edge of this enhancement (second from right) has a similar motion. However, the leading and trailing edges of the more minor enhancement in magnetosheath density show motion towards dusk and back out towards the magnetopause. Note that the positions of the boundaries on the $M$ axis are scaled according to the times of their observation, in order to represent an approximate spatial separation of these structures. The inferred motion is consistent with the entire structure having a scale size of $\sim 0.8 R_{E}$ and moving duskwards across the magnetopause at $\sim 70 \mathrm{~km} \mathrm{~s}^{-1}$.

horizontal line, and the boundary between the open flux tube in the FTE and the closed flux tubes of the magnetosphere proper is represented by the solid curved line marked OCB at each end. The dotted horizontal arrowed lines show the relative trajectories of the Cluster spacecraft through this structure. $\mathrm{C} 1, \mathrm{C} 2$ and $\mathrm{C} 3$ pass closest to the magnetopause, with $\mathrm{C} 2$ leading the other two in this projection. C4 cuts through the structure at a greater distance from the magnetopause.

In the first event, corresponding to the configuration shown in Fig. 9a, the observation of an enhancement of magnetospheric electron density and magnetic field strength prior to the arrival of the magnetosheath electrons suggests that this feature may be on closed magnetospheric field lines that are compressed as the FTE structure advances into them. These are thus indicated on the figure as the hatched region, marked A, to the right of the OCB. Following this enhancement, the spacecraft observe a dropout of the magnetospheric electrons and a rapid enhancement of magnetosheath electrons. This signature is assumed to be associated with the crossing of the OCB and movement onto the oldest reconnected field lines, marked B, within the FTE, i.e. those that have emptied their magnetospheric electrons and have had enough time to refill with magnetosheath plasma. Note that although the electrons might be expected to be able to travel along the reconnected field lines very quickly, it is likely that the relatively cold, dense electrons crossing the magnetopause from the magnetosheath will be inhibited from running ahead of the 

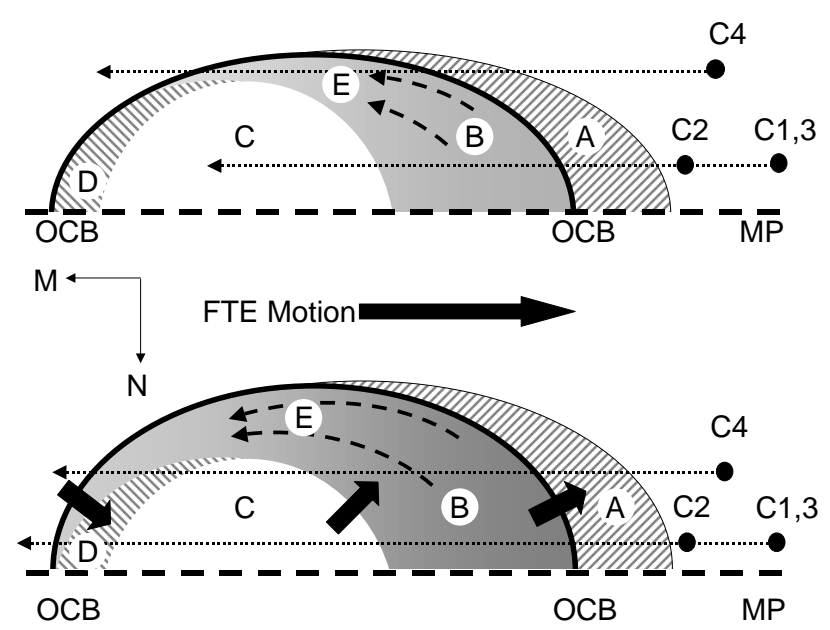

Fig. 9. A possible interpretation of the PEACE observations for the internal structure of FTEs. In each panel, the FTE is assumed to be a bundle of reconnected flux tubes occupying the region between the unperturbed magnetopause (dashed horizontal line marked MP) and an internal boundary between the open and closed flux tubes (represented by the curved solid line marked OCB at each end). The relative trajectories of the 4 spacecraft are represented by the horizontal dotted arrowed lines, which point left as the FTE motion is in the negative $M$ direction, i.e. to the right. The observation of enhanced field strengths and densities of magnetospheric electrons prior to the arrival of the magnetosheath electrons implies a compressed region (marked $\mathrm{A}$ ) on the closed flux tubes ahead of the FTE. On crossing the OCB, the spacecraft will observe a sudden enhancement of magnetosheath electrons on the oldest reconnected field lines (marked B). On moving further into the FTE, the spacecraft move onto more recently reconnected field lines, which may not yet have completely filled with magnetosheath plasma, and thus observe a declining density (marked C). It appears that an enhancement in magnetospheric electrons occurs in the trailing edge of the FTE (marked D). In order to explain the delayed and extended signature at $\mathrm{C} 4$, it is necessary to account for magnetosheath electrons in the region marked $\mathrm{E}$ in the figures. We infer a re-circulation of the oldest reconnected flux tubes along the leading edge of the FTE structure, as indicated by the dashed arrowed lines. During the first event, represented by the upper figure, we suggest that $\mathrm{C} 4$ passes through this region, then out across the OCB and back onto closed field lines and thus does not observe the magnetospheric population marked D. During the second event studied in this paper, represented in the lower figure, we infer that $\mathrm{C} 4$ passed relatively deeper into the FTE, and that the re-circulation of the oldest flux tubes had proceeded sufficiently to push some of these flux tubes as far back as the trailing edge of the FTE. Therefore, all 4 spacecraft observe a second enhancement of magnetosheath electrons after the magnetospheric population (D). In addition, this interpretation is consistent with the inferred motion of the 4 boundaries shown in Fig. 8, as indicated by the heavy arrows in the lower figure.

magnetosheath ions by the requirements of charge neutrality (Burch, 1985; Topliss et al., 2001). The reconnected flux tubes should thus fill with magnetosheath plasma on an ion time scale. We invoke this as an explanation for the more gradual decline in the magnetosheath electron density; as the spacecraft move to more recently reconnected field lines, i.e. towards the left of the FTE region shown in the figure, there has been less time for the flux tubes to fill with magnetosheath plasma. Towards the trailing edge of the FTE (region marked C), there may be essentially no plasma of magnetosheath origin on the observed part of the reconnected flux tubes. Indeed, the most recent reconnected field lines may not have yet emptied their magnetospheric electrons. However, the observation of enhanced fluxes of these electrons on the trailing edge of the FTE implies some local trapping mechanism (e.g. Cowley and Lewis, 1990) to delay the evacuation of these electrons if they are located on the open FTE field lines, as indicated by the hatching marked D on the figure.

The delayed and less structured signature observed at $\mathrm{C} 4$, deeper into the magnetosheath, has strong implications for the structure of the FTE. In order to account for these observations, there must also be some variation in structure in the direction normal to the magnetopause. Note that in a simple picture of a cylindrical FTE filled with magnetosheath plasma, which convects over this configuration of the quartet, we would expect to see a shorter signature at $\mathrm{C} 4$, nested between the signatures observed at $\mathrm{C} 2$, and $\mathrm{C} 1$ and $\mathrm{C} 3$. (We note in passing that this would also be true of planar magnetopause motion back and forth across the quartet, or of a boundary wave causing a brief exit of the quartet into the magnetosheath, suggested as causes of the FTE signature by Hall et al. (1990) and Sibeck (1990, 1992)). Instead, we see the opposite signature during the first event, in that it is extended at $\mathrm{C} 4$ and delayed with respect to the corresponding signatures at the 3 spacecraft closer to the magnetopause. This implies that the cross section of this intrusion of magnetosheath electrons is shaped like the top half of a crescent, or a dolphin dorsal fin. We suggest that there is some recirculation or redistribution of reconnected flux tubes within the FTE, as indicated by the dashed arrowed lines, perhaps as a result of some form of drag operating at the boundary between open and closed flux tubes. We suggest that this results in some of the older reconnected flux tubes containing magnetosheath electrons being pulled back from the leading edge of the FTE and along the Earthward boundary into the region marked $\mathrm{E}$ in the figure. This region of magnetosheath electrons thus lags behind the similar observations seen closer to the magnetopause, and can also be longer in duration and may be a weaker and less structured enhancement.

Support for the above scenario is provided by the more quantitative results obtained from the second event, as indicated in Fig. 9b. In this picture, we have assumed that the re-circulation of older reconnected flux tubes has proceeded far enough for these flux tubes to be observed on the trailing edge of the FTE. Assuming also that the quartet passes through this structure relatively closer to the magnetopause edge than during the first example, such a structure provides normals to the leading and trailing edges of the regions of magnetosheath electrons which are consistent with those observed, as indicated by the solid dark arrows. Although we are not able to determine the cause of this inferred substructure, the above picture is consistent with the timing of the 
PEACE observations and boundary motions. Alternate interpretations of this data must, therefore, also account for this distribution of magnetosheath electrons within the FTE structure.

\section{Summary and conclusions}

We have presented preliminary observations of electrons during a series of magnetospheric FTEs observed by the Cluster PEACE instruments on 2 February 2001, together with the associated FGM data. The signatures observed by the individual spacecraft are consistent in structure and inter-event timing with the FTE interpretation. Differences in the signatures of individual FTEs observed at the 4 spacecraft indicate that there are structures within the FTE with spatial scales of the order or less than the spacecraft separation $(\sim 600 \mathrm{~km})$. Examination of the timings of the signatures at each of the 4 spacecraft shows that these are neither nested signatures associated with the backward and forward motion of a planar section of the magnetopause itself, nor are they consistent with a boundary wave passing around the magnetopause and over the spacecraft locations. Rather, the main enhancements in magnetosheath-like electrons have both a leading and trailing edge which are moving duskwards and into the magnetosphere. Only in the second event we do observe evidence of an electron structure moving back out towards the magnetopause. The timings in this event suggest a structure approximately aligned with the magnetospheric field, with a size of the order of $0.8 R_{E}$ and moving around the magnetopause from noon towards dusk at speeds of the order of $70 \mathrm{~km} \mathrm{~s}^{-1}$. Again, this is consistent with a bundle of reconnected flux tubes receding from a reconnection line and convecting around the magnetopause. We have put forward a possible interpretation of the differences in signatures between the 4 spacecraft. We suggest that the internal structure of the FTE contains a leading edge of the oldest reconnected flux tubes, which shows the abrupt onset of magnetosheath electrons as the spacecraft pass onto these flux tubes. As the FTE passes over the spacecraft location, the spacecraft encounter more recently reconnected field lines with a lower, and eventually vanishing flux of magnetosheath electrons. In order to explain the variation in signatures in the direction normal to the magnetopause, we suggest that some recirculation of the flux tubes within the FTE occurs, perhaps as a result of some form of drag on the flux tube at the leading edge of the FTE. This results in a population of the older reconnected flux tubes, and the associated magnetosheath electrons, extending along the Earthward edge of the FTE and extending even to the trailing edge in the second event. Finally, we note the occurrence of enhanced fluxes of magnetospheric electrons and slightly elevated field strengths ahead of the leading edge of the event, perhaps caused by a "snowplow"like compression of the closed magnetospheric flux tubes as the FTE approaches. A similar enhancement of electrons is also seen towards the rear of the event. This population may be on the most recently reconnected field lines, assuming that some local trapping prevents the almost instantaneous evacuation of these electrons from the field line. Clearly, these suggestions need to be verified by the study of a wider database of such events, and the use of higher order moment data from PEACE and data from other Cluster instruments.

Acknowledgements. During the course of the work, CJO was supported by a United Kingdom PPARC Advanced Fellowship. We acknowledge the efforts of the UCL/MSSL technical and engineering staff in rebuilding the PEACE instruments. We thank the referees for helpful comments.

Topical Editor G. Chanteur thanks G. Paschmann and another referee for their help in evaluating this paper.

\section{References}

Balogh, A., Dunlop, M. W., Cowley, S. W. H., Southwood, D. J., Thomlinson, J. G., Glassmeier, K. H., Musmann, G., Lühr, H., Buchert, S., Acuña, M. H., Fairfield, D. H., Slavin, J. A., Riedler, W., Schwingenschuh, K., Kivelson, M. G., and the Cluster magnetometer team: The Cluster magnetic field investigation, Space Sci. Rev., 79, 65-91, 1997.

Berchem, J. and Russell, C. T.: Flux-transfer events on the magnetopause - spatial-distribution and controlling factors, J. Geophys. Res., 89, 6689, 1984.

Burch, J. L.: Quasi-neutrality in the polar cusps, Geophys. Res. Lett., 12, 469, 1985.

Cowley, S. W. H. and Lewis, Z. V.: Magnetic trapping of energetic particles on open dayside boundary layer flux tubes, Planet. Space Sci., 38, 1343, 1990.

Crooker, N. U. and Siscoe, G. L.: On mapping flux-transfer events to the ionosphere, J. Geophys. Res., 95, 3795, 1990.

Daly, P. W., Williams, D. J., Russell, C. T., and Keppler, E.: Particle signature of magnetic-flux transfer events at the magnetopause, J. Geophys. Res., 86, 1628, 1981.

Daly, P. W., Saunders, M. A., Rijnbeek, R. P., Sckopke N., and Russell, C. T.: The distribution of reconnection geometry in fluxtransfer events using energetic ion, plasma and magnetic data, J. Geophys. Res., 89, 3843, 1984.

Dungey, J. W.: Interplanetary magnetic field and the auroral zones, Phys. Rev. Lett., 6, 47, 1961.

Elphic, R. C. and Southwood, D. J.: Simultaneous measurements of the magnetopause and flux-transfer events at widely separated sites by AMPTE UKS and ISEE-1 and ISEE-2, J. Geophys. Res., 92, $13666,1987$.

Elphic, R. C., Lockwood, M., Cowley S. W. H., and Sandholt, P. E.: Flux-transfer events at the magnetopause and in the ionosphere, Geophys. Res. Lett., 17, 2241, 1990.

Elphic, R. C., Baumjohann, W., Cattell, C. A., Lühr, H., and Smith, M. F.: A search for upstream pressure pulses associated with flux-transfer events - an AMPTE/ISEE case-study, J. Geophys. Res., 99, 13 521, 1994.

Fairfield, D. H.: Average and unusual locations of the Earths magnetopause and bow shock, J. Geophys. Res., 76, 6700, 1971.

Farrugia, C. J., Southwood, D. J., Cowley, S. W. H., Rijnbeek, R. P., and Daly, P. W.: 2-Regime flux-transfer events, Planet Space Sci., 35, 737, 1987.

Farrugia, C. J., et al.: A multi-instrument study of flux transfer event structure, J. Geophys. Res., 93, 14 465, 1988.

Goertz, C. K., Nielsen, E., Korth, A., Glassmeier, K. H., Haldoupis, C., Hoeg P., and Hayward, D.: Observations of a possible ground 
signature of flux-transfer events, J. Geophys. Res., 90, 4069, 1985.

Hall, D. S., Chaloner, C. P., Bryant, D. A., Lepine, D. R., and Tritakis, V. P.: Electrons in the boundary-layers near the dayside magnetopause, J. Geophys. Res., 96, 7869, 1991

Hesse, M., Birn J., and Schindler, K.: On the topology of fluxtransfer events, J. Geophys. Res. 95, 6549, 1990.

Hubert, D., Lacombe, C., Harvey, C. C., Mangeney, A., and Russell, C. T.: Density and field structure of a FTE observed in the magnetosphere, Geophys. Res. Lett., 19, 965, 1992.

Jacob, J.D. and Cattell, C.: High time resolution measurements of upstream magnetic-field and plasma conditions during fluxtransfer events at the Earths dayside magnetopause, Geophys. Res. Lett., 20, 2007, 1993.

Kan, J. R.: A theory of patchy and intermittent reconnections for magnetospheric flux-transfer events, J. Geophys. Res., 93, 5613, 1988.

Kawano, H. and Russell, C. T.: Survey of flux transfer events observed with the ISEE 1 spacecraft: Rotational polarity and the source region, J. Geophys. Res., 101, 27 299, 1996.

Kawano, H. and Russell, C. T.: Survey of flux transfer events observed with the ISEE 1 spacecraft: Dependence on the interplanetary magnetic field, J. Geophys. Res., 102, $11307,1997$.

Klumpar, D. M., Fuselier, S. A., and Shelley, E. G.: Ion composition measurements within magnetospheric flux-transfer events, Geophys. Res. Lett., 17, 2305, 1990.

Ku, H. C. and Sibeck, D. G.: Internal structure of flux transfer events produced by the onset of merging at a single $X$ line, $\mathrm{J}$. Geophys. Res., 102, 2243, 1997.

Kuo, H., Russell, C. T., and Le, G.: Statistical studies of fluxtransfer events, J. Geophys. Res., 100, 3513, 1995.

Le, G., Russell, C. T., and Kuo, H.: Flux-transfer events - spontaneous or driven?, Geophys. Res. Lett., 20, 791, 1993.

Le, G., Gosling, J. T., Russell, C. T., Elphic, R. C., Thomsen, M. F., and Newbury, J. A.: The magnetic and plasma structure of flux transfer events, J. Geophys. Res., 104, 233, 1999.

Lee, L. C., Ma, Z. W., Fu, Z. F., and Otto, A.: Topology of magnetic-flux ropes and formation of fossil flux-transfer events and boundary-layer plasmas, J. Geophys. Res., 98, 3943, 1993.

Lockwood, M., Cowley, S. W. H., Sandholt, P. E., and Lepping, R. P.: The ionospheric signatures of flux-transfer events and solarwind dynamic pressure changes, J. Geophys. Res., 95, 17113, 1990.

Lockwood, M.: Flux-transfer events at the dayside magnetopause - transient reconnection or magnetosheath dynamic pressure pulses?, J. Geophys. Res., 96, 5497, 1991.

Lockwood, M. and Wild, M. N.: On the quasi-periodic nature of magnetopause flux-transfer events, J. Geophys. Res., 98, 5935, 1993.

Nakamura, M. and Scholer, M.: Structure of the magnetopause reconnection layer and of flux transfer events: Ion kinetic effects, J. Geophys. Res., 105, 23 179, 2000.

Oieroset, M., Lühr, H., Moen, J., Moretto T., and Sandholt, P. E.: Dynamical auroral morphology in relation to ionospheric plasma convection and geomagnetic activity: Signatures of magnetopause $X$ line dynamics and flux transfer events, J. Geophys. Res., 101, 13 275, 1996.

Paschmann, G., et al.: Plasma acceleration at the Earths magnetopause: Evidence for reconnection, Nature, 282, 243, 1979.

Paschmann, G., Haerendel, G., Papamastorakis, I., Sckopke, N., Bame, S. J., Gosling, J. T., and Russell, C. T.: Plasma and magnetic-field characteristics of magnetic-flux transfer events, J.
Geophys. Res., 87, 2159, 1982.

Provan, G., Yeoman, T. K., and Milan, S. E.: CUTLASS Finland radar observations of the ionospheric signatures of flux transfer events and the resulting plasma flows, Ann. Geophysicae, 16, 1411, 1998.

Rijnbeek, R. P., Cowley, S. W. H., Southwood, D. J., and Russell, C. T.: Observations of reverse polarity flux-transfer events at the earths dayside magnetopause, Nature, 300, 23, 1982.

Rijnbeek, R. P., Cowley, S. W. H., Southwood, D. J., and Russell, C. T.: A survey of dayside flux- transfer events observed by ISEE-1 and ISEE-2 magnetometers, J. Geophys. Res., 89, 786, 1984.

Rijnbeek, R. P., et al.: A magnetic boundary signature within flux transfer events, Planet. Space Sci., 25, 871, 1988.

Rodger, A. S. and Pinnock, M.: The ionospheric response to flux transfer events: The first few minutes, Ann. Geophysicae, 15, 685, 1997.

Russell, C. T. and Elphic, R. C.: Initial ISEE magnetometer results: Magnetopause observations, Space Sci. Rev., 22, 681, 1978.

Russell, C. T., Le, G., and Kuo, H.: The occurrence rate of flux transfer, Adv. Space Res., 18, 197, 1995.

Saunders, M. A., Russell, C. T., and Sckopke, N.: Flux-transfer events - scale size and interior structure, Geophys. Res. Lett., 11, 131, 1984.

Scholer, M., Hovestadt, D., Ipavich, F. M., and Gloeckler, G.: Energetic protons, alpha-particles, and electrons in magnetic-flux transfer events, J. Geophys. Res., 87, 2169, 1982.

Sibeck, D. G.: Transient events in the outer magnetosphere boundary waves or flux-transfer events?, J. Geophys. Res., 97, 4009, 1992.

Sibeck, D. G. and Siscoe, G. L.: Downstream properties of magnetic-flux transfer events, J. Geophys. Res., 89, 709, 1984.

Sibeck, D. G. and Smith, M. F.: Magnetospheric plasma flows associated with boundary waves and flux-transfer events, Geophys. Res. Lett., 19, 1903, 1992.

Sibeck, D. G., et al.: A model for the transient magnetospheric response to sudden solar wind dynamic pressure variations, J. Geophys. Res., 95, 3755, 1990.

Smith, M. F. and Owen, C. J.: Temperature anisotropies in a magnetospheric FTE, Geophys. Res. Lett., 19, 1907, 1992.

Song, P., Le, G., and Russell, C. T.: Observational differences between flux-transfer events and surface-waves at the magnetopause, J. Geophys. Res., 99, 2309, 1994.

Sonnerup, B. U. Ö., et al., Evidence for magnetic reconnection at the Earths magnetopause, J. Geophys. Res., 86, 10 049, 1981.

Sonnerup, B. U. Ö.: On the stress balance in flux-transfer events, J. Geophys. Res., 92, 8613, 1987.

Southwood, D. J.: The ionospheric signature of flux-transfer events, J. Geophys. Res., 92, 3207, 1987.

Southwood, D. J., Saunders, M. A., Dunlop, M. W., Mierjedrzejowicz, W. A. C., and Rijnbeek, R. P.: A survey of flux-transfer events recorded by the UKS spacecraft magnetometer, Planet Space Sci., 34, 1349, 1986.

Southwood, D. J., Farrugia, C. J., and Saunders, M. A.: What are flux-transfer events?, Planet Space Sci., 36, 503, 1988.

Thomsen, M. F., Stansberry, J. A., Bame, S. J., Fuselier, S. A., and Gosling, J. T.: Ion and electron velocity distributions within fluxtransfer events, J. Geophys. Res., 92, 12 127, 1987.

Topliss, S., Johnstone, A. D., Coates, A. J., Peterson, W. K., Kletzing, C. A., and Russell, C. T.: Charge neutrality and ion conic distributions at the equatorward electron edge of the mid-altitude cusp, J. Geophys. Res., 106, 21 095, 2001. 